\title{
Low Buffering and Waiting-Time Video-on-Demand Broadcasting Scheme for WiMAX Systems
}

\author{
Jenhui Chen \\ Department of Computer Science and \\ Information Engineering \\ Chang Gung University \\ Kweishan, Taoyuan, Taiwan, R.O.C. \\ jhchen@mail.cgu.edu.tw
}

\author{
Jui-Hsiang Sun \\ Department of Computer Science and \\ Information Engineering \\ Chang Gung University \\ Kweishan, Taoyuan, Taiwan, R.O.C. \\ sun.semmer@gmail.com
}

\begin{abstract}
Providing video-on-demand (VoD) services for mobile devices in next generation networks such as the worldwide interoperability for microwave access (WiMAX) is an important trend. These multimedia services may require mobile devices keeping a large buffer size for video playing and let mobile devices spend a lot of time for waiting the start of the requested video. To improve this drawback, many mechanisms such as reverse fast broadcasting (RFB) scheme and division-base broadcasting (DBB) scheme are proposed to overcome problems of lowering the buffer size and shortening the waiting time for video playing. However, the required buffer size and waiting time are still not acceptable for wireless environment due to the limited buffer size and mobility consideration of the mobile devices. Thus we first propose a proactive scheme named remainder arrangement broadcasting (RAB) to lower the required buffer size. Then, to shorten the start time of video playing, we add the reactive method to RAB scheme named hybrid RAB (HRAB). Simulation results show that RAB will achieve a lower buffer size, which is under $10 \%$, without requiring large bandwidth and HRAB will shorten the waiting time well.
\end{abstract}

\section{Keywords}

algorithm, broadcasting, buffer, VoD, waiting time, wireless, WiMAX

\section{INTRODUCTION}

VoD applications have been very popular in recent years. These services are delay sensitive and require a lot of bandwidth and disk spaces. It is a very challenging problem

\footnotetext{
*Correspondence should be addressed to J. Chen. This work was supported by the National Science Council, Taiwan, R.O.C., under Contract NSC95-2221-E-182-023.
}

\footnotetext{
Permission to make digital or hard copies of all or part of this work for personal or classroom use is granted without fee provided that copies are not made or distributed for profit or commercial advantage and that copies bear this notice and the full citation on the first page. To copy otherwise, to republish, to post on servers or to redistribute to lists, requires prior specific permission and/or a fee. MOBIMEDIA 2007, August 27-29, Nafpaktos, Greece Copyright $\odot 2007$ ICST 978-963-06-2670-5 DOI 10.4108/ICST.MOBIMEDIA2007.1902
}

that provides such services for mobile devices in wireless environment. The VoD broadcasting system is a typical client-server architecture and can be divided into to parts: proactive $[1,2,3,4,5,6,7,8]$ and reactive $[9,10]$ schemes. In the proactive scheme, a video is split up into a number of segments with the same size, which will be periodically broadcasted at the assigned channel. In the reactive scheme, subscribers may request the server for ordering a video at any time, and then the server will classify the similar requesting time of subscribers into a batch. In the meantime the server broadcasts (or multicasts) the required video at an assigned channel. The 802.16 standard [11, 12] can provide high-speed wireless access in metropolitan area network (WMAN) and contain the characteristics of broadband, seamless handover, quality-of-service (QoS), and high transmission rate in all-IP environment, which makes it suitable for mobile multimedia transmission platform.

In the previous studies, the VoD system focuses on diminishing waiting time and buffer requirement. In the proactive $\mathrm{VoD}$, the maximum waiting time is the transmission time of a segment. If a large number of segments has been periodically broadcasted at the assigned channels, it will reduce both the waiting time and the huge bandwidth. Because of the limited resource, the system can not allocate too many channels to broadcast a large number of segments in wireless network. Most of the time, transmitting is only allowed at a single channel in a wireless network [2]. For this reason, there is a problem of huge waiting time which equals to $2-20 \%$ of a video length in video transmission of wireless network. In reverse fast broadcasting (RFB) [1], subscribers need to buffer $25-33 \%$ of a video. If a 120 minutes video is playing at a $1 \mathrm{Mbps}$ rate, the video size will be 900 Mbytes, so 225-270 Mbytes of buffering and 2-24 mins of waiting time will be required. For mobile subscriber station (MSS) the loading space is limited and the huge buffering will not be suitable in wireless environment.

In this paper, two methods are proposed to reduce the buffering requirement and the waiting time of subscribers on the WiMAX-VoD system. In order to achieve the minimum buffering requirement, the RAB scheme is proposed to rearrange the order of the segments so they can continuously be played one by one. On the other hand, in order to shorten the subscriber's waiting time, the HRAB scheme is proposed to transfer segments under reactive scheme and higher speed transmission in the architecture of adaptive 


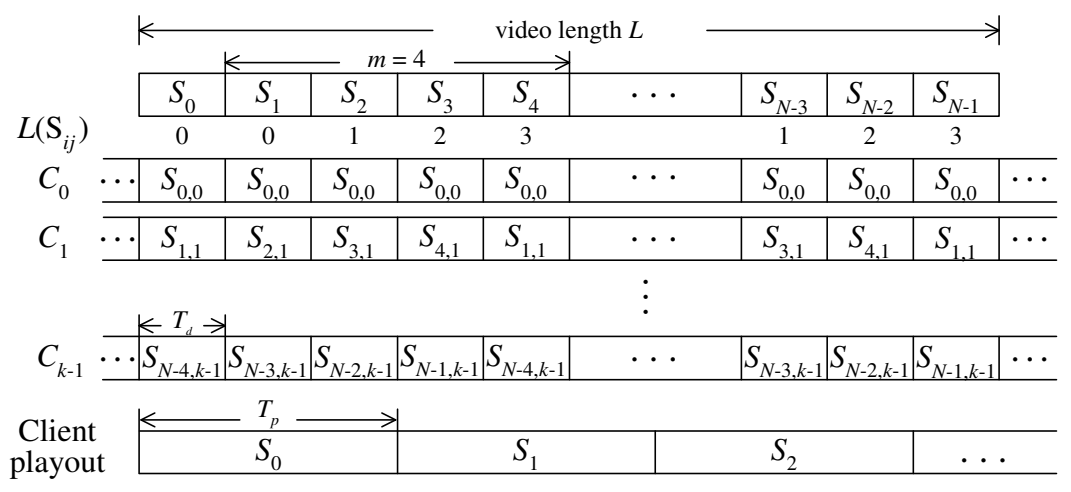

(a)

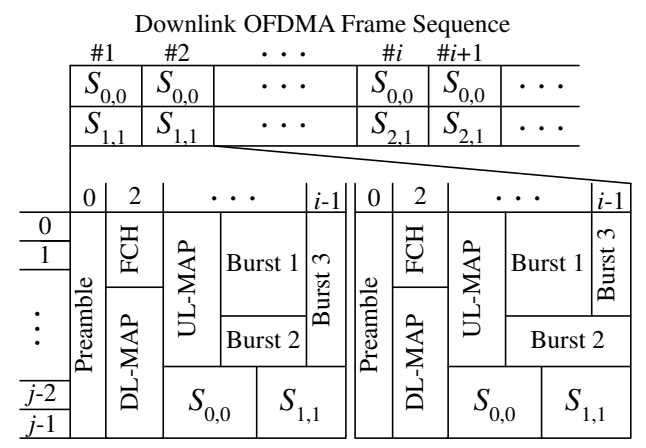

$k$ Downlink Frame $\# 1 \rightarrow \mid k$ Downlink Frame \#2 $\rightarrow$

(b)

Figure 1: An illustration of the VoD system in WiMAX system; (a) the VoD program (b) the OFDMA downlink frame structure.

modulation and coding (AMC), thus the waiting time will be greatly reduced.

The outline of this paper is as follows. Section 2 describes the system model; Section 3 provides RAB and HRAB schemes in detail; Section 4 describes our implementation of proposed scheme results comparing proposed scheme performance with RFB and DBB schemes. Finally, the conclusion and the future works are discussed in Section 5.

\section{SYSTEM MODEL}

To evaluate the performance of the video broadcast scheme, a VoD system model is constructed first. Suppose that the VoD broadcasting system will periodically broadcast a video film of size $L$ for supporting VoD services to the subscribers. For the reason of successful transmission possibility, the video film will be divided into $N$ independent segments denoted by $\mathbb{S}=\left\{S_{0}, S_{1}, \ldots, S_{N-1}\right\}$, where $\mathbb{S}$ represents the set of these segments. The size of segment $i$ denoted as $\left|S_{i}\right|=L / N$ is assumed equal. These segments are scheduled and broadcasted at $k$ independent channels denoted as $\mathbb{C}=\left\{C_{0}, C_{1}, \ldots, C_{k-1}\right\}$ as shown in Figure 1 . In order to service all subscribers, the data rate of each channel is transmitted in the basic rate denoted as $D_{b}$ and the total needed bandwidth for broadcasting the video film is $k D_{b}$. Assume each subscriber needs a video playing rate $P_{r}$ to play a video and it satisfies the condition $P_{r} \leq D_{b}$, which means that the download rate should be faster than the play rate so that the subscriber can obtain enough data for process. This proposition is called the playback ratio $\alpha$ and is given by

$$
\alpha=\frac{D_{b}}{P_{r}} \geq 1 .
$$

According to $\alpha$, the VoD broadcasting system can determine a tolerant time period for the download subscribers that subscribers can just play the needed segment from their buffer if they have downloaded a $S_{i}$ from the server. Since, in proactive scheme, the first segment $S_{0}$ should be repeated in channel $C_{0}$ for a new entry, the tolerant time period of each $C_{i}$ will be

$$
\left\{\begin{array}{ll}
m=1, & \text { if } C_{0} \\
m=\lfloor\alpha\rfloor+1, & \text { otherwise }
\end{array},\right.
$$

where $m$ represents the number of segments in one period.
Let $S_{i j}$ represent $S_{i}$ allocated in $C_{j}$ and the group of segments allocated in $C_{j}$ be $\mathbb{S}_{j}=\left\{S_{i j}\right\}$, where $i=m j-\lfloor\alpha\rfloor+$ $1, \ldots, m j$ and $j=1, \ldots, k-1$. That is, $\mathbb{S}$ is partitioned into $\left\{\mathbb{S}_{0}, \mathbb{S}_{1}, \ldots, \mathbb{S}_{k-1}\right\}=\bigcup_{i \in I} \mathbb{S}_{i}$, where $I$ is an index set, and allocated into $k$ channels as shown in Figure 1.

Thus, based on $k$ and $m$, one video film can be divided into $N$ equal size segments and given by

$$
\begin{aligned}
N & =(k-1) m+1 \\
& =(k-1)(\lfloor\alpha\rfloor+1)+1 \\
& =k\lfloor\alpha\rfloor+k-\lfloor\alpha\rfloor-1+1 \\
& =k m-\lfloor\alpha\rfloor .
\end{aligned}
$$

Notice that, for each new entry, i.e., the new subscriber of the VoD broadcasting, it will spend a waiting time $T_{w} \leq$ $\left|S_{i}\right| / D_{b}$ for getting start of video playing whenever they enter the system. In this paper, we assume that $S_{i}$ can be played immediately as soon as it is being downloaded. The location of $S_{i j}$ denoted as $L\left(S_{i j}\right)$ is used to indicate the $S_{i}$ used to broadcast in $C_{j}$, where $0 \leq L\left(S_{i j}\right) \leq m-1$ and segments in $C_{j}$ are from $S_{(m j-\lfloor\alpha\rfloor+1) j}$ to $S_{(m j) j}$, where $j \in[1, k-1]$.

The playback time $T_{p}$, which is defined as the time the subscriber needs to play a segment, can be calculated as $T_{p}=\left|S_{i}\right| / P_{r}$. The download time $T_{d}$ represents the required time that a segment is downloaded from the server to the subscriber and is equal to $T_{d}=\left|S_{i}\right| / D_{b}$. Thus the playback ratio can also be represented as $\alpha=T_{p} / T_{d}$ and is subject to $T_{d} \leq T_{p}$. Let $t_{d}$ and $t_{s}$ denote the time that the subscriber demands the service and the time that the subscriber starts to download the first segment. Thus $T_{w}=t_{s}-t_{d}, 0 \leq T_{w} \leq$ $T_{d}$.

Let $t_{c i}$ denote the starting time of $S_{i j}$ first appears after the demand of the subscriber, $t_{n i}$ represent the starting time of the next $S_{i j}$ appears and $t_{n i}=\left(t_{c i}+m T_{d}\right)$, and $t_{u i}$ denote the time the subscriber needs to demand the segment $S_{i j}$, where $t_{n i} \leq t_{u i}$. In the reactive scheme, let $\gamma$ and $\rho$ be the time the server prepares a video film for downloading and the time the subscriber spends for waiting services, respectively. The waiting time $\rho$ satisfies $\gamma \leq \rho \leq T_{d}$. Thus, the user tolerable waiting time $T_{w}$ is given below

$$
\left\{\begin{array}{ll}
0 \leq T_{w} \leq T_{d}, & \text { proactive scheme } \\
\gamma \leq T_{w} \leq \rho, & \text { reactive scheme }
\end{array} .\right.
$$


Assume a subscriber needs a minimum required buffer size $\beta(t)$ to store the downloaded file for playing. The buffer size can be calculated as

$$
\begin{aligned}
\beta(t) & =t\left(n D_{b}-P_{r}\right) \\
& =t\left(n \alpha P_{r}-P_{r}\right) \\
& =t P_{r}(n \alpha-1),
\end{aligned}
$$

where $n$ denotes as the number of unplayed segments stored in the buffer during the time interval $t$. Let $D_{h}$ and $\lfloor\alpha\rfloor^{\prime}$ represent the higher channel transmission rate and playback ratio, respectively. The ratio between original transmission rate is $D_{h} / D_{b}=\lfloor\alpha\rfloor^{\prime} /\lfloor\alpha\rfloor=\sigma$; The segment downloading time is $T_{d}^{\prime}=T_{p} /\lfloor\alpha\rfloor^{\prime}$.

In the system model, the environment is assumed that consists of the following qualities: 1)The subscribers can bring up their demands anytime and download while playing the film when the next $S_{00}$ occurs; 2)The subscriber can download multi-channels' data at the same time; 3)Breaking off is not available when the subscriber is playing films; 4)The subscriber can start playing data as soon as it starts receiving or downloading; 5)The subscriber has its own buffer and it can receive and store new data while playing data; 6)The buffer has enough capacity to store the data; 7)The subscriber starts receiving broadcast data after it demands playing the data.

\section{PROPOSED SCHEMES}

The transmission system considered in this paper is the IEEE 802.16-2004 standard and the physical (PHY) layer uses orthogonal frequency division multiple access with time division duplex (OFDMA/TDD) scheme for data transmissions. Two channels are used to broadcast video and films ready for broadcasting will be divided into similar sizes at downloading frame and broadcasted cyclically by modulation intensity of the minimum modulation at two virtual VoD channels.

These two virtual VoD channels are constructed by OFDMA burst that also interpret VoD burst's information at DLMAP. In this system, only two channels are used with the lowest transmission modulation for servicing most subscribers. $D_{b}$ uses QPSK-1/2 modulation's transmission rate in WiMAX. For example, QPSK-1/2's data rate $\sim 3$ Mbps; a MPEG1encoded playback rate $\sim 1 \mathrm{Mbps},\lfloor\alpha\rfloor=3 \mathrm{Mbps} / 1 \mathrm{Mbps}$ $=3$. There are still more encoded formats at the format of video, such as MPEG2, MPEG4, H.264, RM (real media) $[15,16]$. In addition, the audio can also be used in this type of application, like moving picture experts group audio Layer3 (MP3)-encoded audio which has a playback ratio of $128 \mathrm{Kbps}$. In this paper, the $\lfloor\alpha\rfloor$ as integer and larger than 1. By this method the leftover data in the buffer can be broadcasted when MSS is under interference with abnormal video playback or processing handover.

From the framework mentioned above, a subscriber will meet a large buffering capacity which is a heavy burden to the mobile devices. The buffering requirement can be greatly reduced after adding $\mathrm{RFB}$, but segments will still continue to download when $t_{n i} \geq t_{u i}$. However the waiting time is still too long for subscriber with a single channel in wireless. Therefore, the RAB and the HRAB are proposed to the problems of buffer requirement and waiting time.

\subsection{RAB Scheme}

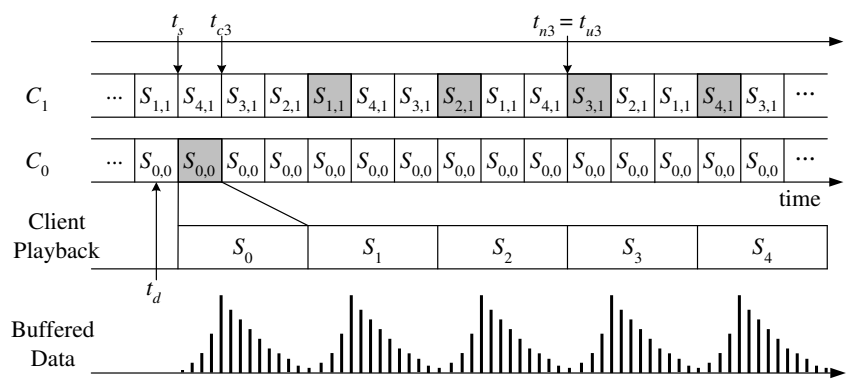

Figure 2: An illustration of segment downloading of RAB when $m=4$.

Generally the order of the broadcasting scheme is assembled according to the order of the segment. Different channels will have different segments placed. Suppose there are $k$ channels, the segment numbering on the $i$ channel $C_{j}$ will be $2^{i-1}$ to $2^{i}-1$, but it will be downloading multi-segments at the same time. In order to minimize the buffer requirement, a new segment will be played closely after the previous segment. Such method can be achieved when $t_{n i}=t_{u i}$. In order to reach this goal, the order of segments on a channel must be adjusted.

By using the playback ratio and the order on each channel, the order of a broadcasting period can be recalculated. At the initiation process $S_{i j}, i \in[1, N-1]$, is arranged according to the segment number in the broadcasting period. The server will know the rate of video playback and the smallest rate of transmission in the system, so the $\lfloor\alpha\rfloor, N$ and $m$ can be calculated. The location of $S_{i j}$ in broadcasting period is $L\left(S_{i j}\right)$, and we know the required buffer to play out more than the playing and downloading the film at the same time based on the equation (5). The minimized required-buffer can be achieved as the different time between $S_{i j}$ and $S_{(i+1) j}$, witch is equal to $T_{p}\left(\lfloor\alpha\rfloor T_{d}\right)$. Therefore, we can re-arrange $L\left(S_{i j}\right)$ within broadcasting period to have the $\lfloor\alpha\rfloor T_{d}$ intervals between both $S_{i j}$ and $S_{(i+1) j}$, it can be calculated as

$$
L\left(S_{(i+1) j}\right)=\left(\lfloor\alpha\rfloor+L\left(S_{i j}\right)\right) \bmod m .
$$

The location of first segment in broadcasting period is 0 as initialization, $L\left(S_{(i+1) j}\right)$ is calculated by both $L\left(S_{i j}\right)$ and $\lfloor\alpha\rfloor$ until all locations of segments are re-calculated. If there is 0 after the calculation $L\left(S_{(i+1) j}\right)$, then the $L\left(S_{(i+1) j}\right)$ will be placed on the last position. Algorithm 1 presents the detailed descriptions of scheduling $\mathbb{S}$ to corresponding $\mathbb{C}$. In Figure 2, for example, $\mid \alpha\rfloor=3$ and $m=4$. Therefore, a continuously film will be cut into 5 same sized segments $(N=5)$. $S_{00}$ will be broadcasted at channel 0 , and $S_{11}$ to $S_{41}$ will be broadcasted cyclically at channel 1 . Segments downloaded by a subscriber are colored gray. Below the graph is the subscriber's usage on the capacity of buffer. $L\left(S_{11}\right)$ is equal to zero, $L\left(S_{21}\right)=(3+0) \bmod 4=3$. After the calculation $L\left(S_{11}\right)$ to $L\left(S_{41}\right)$ are $0,3,2,1$, and the location after re-arrangement are $S_{11}, S_{41}, S_{31}, S_{21}$. When subscriber demand the film, it will wait until $d / 2$ 's time over and then start to download $S_{00}$. After that, it will download according to the order of the series $S_{11}$ to $S_{41}$. When a subscriber is downloading the segment, it will follow the algorithm of RFB to make each download correspond to $t_{n i}=t_{u i}$. This will make every $t_{n i}=t_{u i}$ minimized as a 


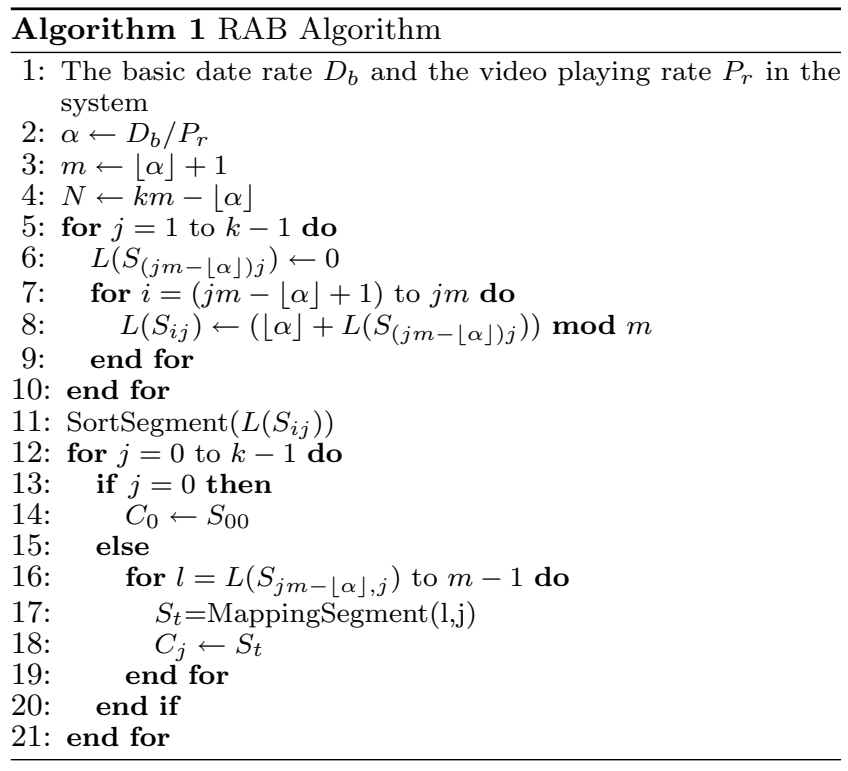

result of the data in each buffering.

\subsection{HRAB Scheme}

On a normal proactive method, the server will frequently broadcast segment $S_{00}$ at channel 0 . This way subscribers can receive the first segment anytime after entering the system. The largest waiting time is equal to the transmission time of a segment $T_{d}$, and the average waiting time is half of $T_{d}$. The length of the waiting time is determined by the number of the segments and the data rate of transmission $T_{d}=\left|S_{i}\right| / D_{b}$. In the proposed scheme, the number of segments is small because the playback ratio $\lfloor\alpha\rfloor$ is used as the parameter of segment. Thus the average waiting time is too long for subscribers. For example, an one hour video clip is deviled into five equal segments, $T_{p}=60 / 5=12 \mathrm{mins}$. Then the transmission time for one segment is $T_{d}=T_{p} /\lfloor\alpha\rfloor=4$ mins, $(\lfloor\alpha\rfloor=3)$, and the average waiting time is $T_{d} / 2=2$ mins. Hence, we use the reactive method to reduce the waiting time.

At tolerate time, when each subscriber requests service from the server, reactive batch scheme can give the same downloaded video to each subscriber after all demand batch and in the AMC scheme is provided within the PHY layer in WiMAX system, so it can supply different modulation on each connection. By using high modulation, the transmission time is shorter than using low modulation and will increase the release of resource soon. Combining the advantage of reactive and the AMC scheme, the waiting time of subscriber will be greatly lowered to the maximum $\rho+\gamma$. The $\delta$ can be calculated as

$$
\left\{\begin{array}{ll}
T_{w}>(\rho+\gamma), & T_{w}=(\rho+\gamma) \\
T_{w} \leq(\rho+\gamma), & 0 \leq T_{w} \leq(\rho+\gamma)
\end{array} .\right.
$$

If $T_{w}>(\rho+\gamma)$, the server will batch the demand within the period after $\rho$ waiting time, and reactively playback segment 0 . If $T_{w} \leq(\rho+\gamma)$, the server will make subscribers wait until the next segment 0 is being broadcasted. Figure 3 assumes $T_{w}>(\rho+\gamma)$, which will be discuss at normal and the worst conditions.

- Normal Case: The subscriber enters the system at

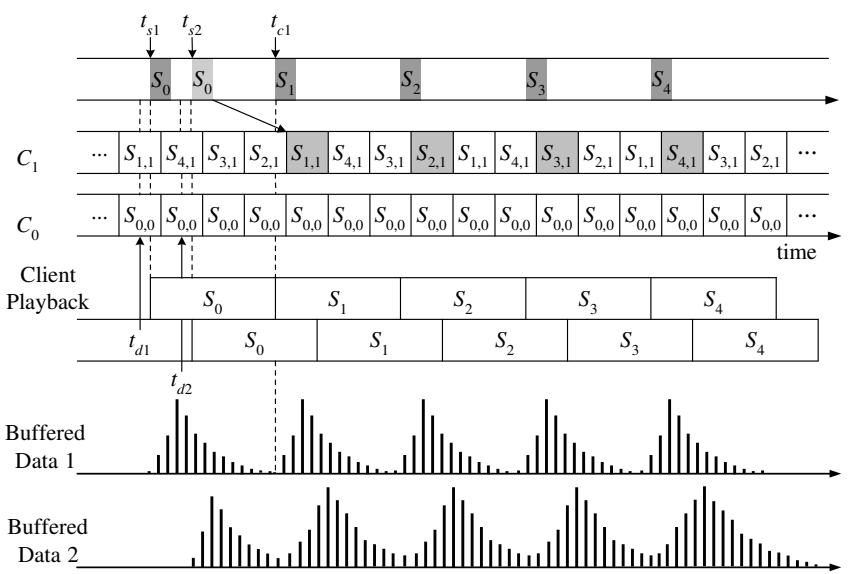

Figure 3: An illustration of segment downloading of HRAB when $m=4$.

time $t_{d 2}$, and provides segment 0 to subscriber with high data rate $D_{b}^{\prime}$ (the maximum tolerable high modulation for subscribers) after waiting time $T_{w} . S_{11}$ can revive right after the $S_{00}$ playback, and then it uses the server broadcasting data to continue receiving.

- Worst Case: The subscriber enters the system at time $t_{d 1}$, and it also provides segment 0 to subscriber with high data rate after waiting time $T_{w}$. However $S_{1}$ will not be received after the playback of $S_{00}$; when the $S_{00}$ finished, the server has to receive the segments coming afterwards. In this case it will can generate more burden to the system comparing with the normal case, but it will only happen on one segment in one broadcasting cycle. Its average appearance probability is $P=1 / m$ plus the tolerable subscriber waiting time $\rho$. Therefore the probability of the worst case is defined as

$$
P_{w}=\frac{1}{m}\left(1-\frac{\rho}{d}\right) .
$$

\section{SIMULATION RESULTS}

The system-specific parameters IEEE 802.16e protocol and the VoD broadcasting system parameters used are shown in Table 1 and Table 2, respectively.

\begin{tabular}{|c|c|}
\hline Parameter & Value \\
\hline Spectrum $(\mathrm{GHz})$ & $2.40-2.46$ \\
\hline Bandwidth $(\mathrm{BW})(\mathrm{MHz})$ & 20 \\
\hline FFT size $\left(N_{\mathrm{FFT}}\right)$ & 2048 \\
\hline $\mathrm{DL} / \mathrm{UL}$ ratio & $3: 2$ \\
\hline OFDMA frame length (ms) & 5 \\
\hline Useful symbol time $\left(T_{b}\right)(\mu \mathrm{s})$ & 91.4 \\
\hline Guard time $\left(T_{g}=T_{b} / 8\right)(\mu \mathrm{s})$ & 11.4 \\
\hline OFDMA symbol time $\left(T_{o}=T_{b}+T_{g}\right)(\mu \mathrm{s})$ & 102.9 \\
\hline No. of subchannels & 60 \\
\hline No. of OFDMA symbol per frame & 48 \\
\hline Avg. time of internet delay $\left(T_{\mathrm{ID}}\right)(\mathrm{ms})$ & 50 \\
\hline Avg. time of frame synchronize ( $\left.T_{\mathrm{SYN}}\right)(\mathrm{ms})$ & 5 \\
\hline $\mathrm{MSS} \leftrightarrows \mathrm{SBS}$ (1frame*2way) (ms) & 10 \\
\hline $\mathrm{SBS} \leftrightarrows \mathrm{TBS}$ (1frame*2way) (ms) & 10 \\
\hline
\end{tabular}

Table 1: Parameters Used in WiMAX

Referring to (5), the needed buffer size of downloading and broadcasting can be calculated, and the download time 


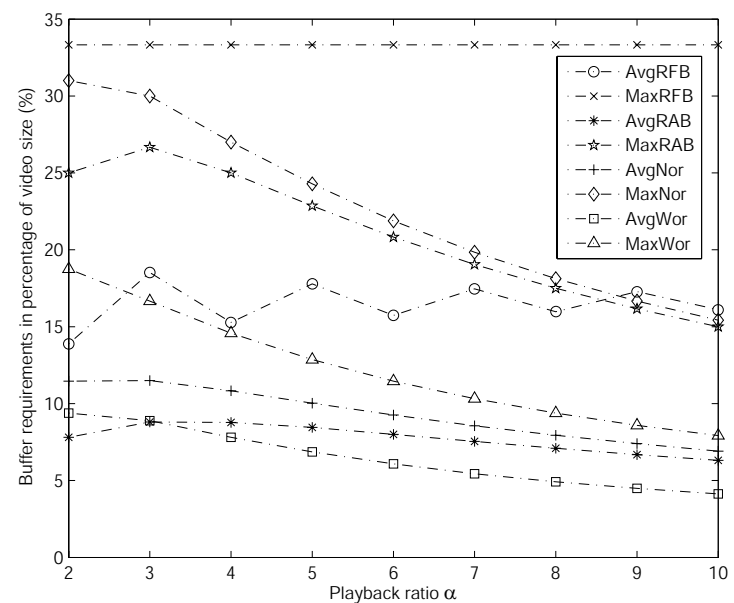

Figure 4: Comparison of required buffers.

Table 2: Parameters Used in VoD

\begin{tabular}{ll}
\hline Parameter & Value \\
\hline \hline Video size $L$ (Mbyte) & 900 \\
Number of channels $k$ & 2 \\
Minimum transmission rate $D_{b}(\mathrm{Mbps})$ & $2-3$ \\
Playback rate $P_{r}(\mathrm{Mbps})$ & $0.3-1$ \\
Playback ratio $\lfloor\alpha\rfloor$ & $2-10$ \\
Broadcasting period $m$ & $3-11$ \\
Number of split video $N$ & $4-12$ \\
Playing a segment time $T_{p}(\mathrm{~s})$ & $1800-600$ \\
Downloading a segment time $T_{d}(\mathrm{~s})$ & $900-60$ \\
Number of downloading segments $n$ & $0-2$ \\
Preparing time of system $\gamma(\mathrm{s})$ & 1 \\
Waiting time that subscribers can tolerate $\rho(\mathrm{s})$ & 10
\end{tabular}

is denoted as $T_{d}$. When the size of a time slot is the downloaded segment time, the total video playing time is $N\lfloor\alpha\rfloor$ slots, and required buffer of each slot has to add up the previous leftover. The first slot can be calculated as

$$
\beta_{\text {slot }}=T_{d} D_{b}(n\lfloor\alpha\rfloor-1),
$$

The $i \in[1, N\lfloor\alpha\rfloor]$ slot is shown as

$$
\beta_{\text {slot }_{i}}=\beta_{\text {slot }_{i-1}}+T_{d} P_{r}(n\lfloor\alpha\rfloor-1),
$$

The total buffer from downloading video and finishing playback will be

$$
\beta_{\text {Total }}=\sum_{i=1}^{N\lfloor\alpha\rfloor} \beta_{\text {slot }_{i}} .
$$

In HRAB scheme, buffer can be calculated as

$$
\begin{aligned}
\beta_{H R A B} & =T_{d}^{\prime} D_{b}\left(n\lfloor\alpha\rfloor^{\prime}-1\right) \\
& =\frac{T_{d}}{\sigma} D_{b}(n \sigma\lfloor\alpha\rfloor-1) \\
& =T_{d} D_{b}\left(n\lfloor\alpha\rfloor-\frac{1}{\sigma}\right) .
\end{aligned}
$$

Figure 4 illustrates the relationship between the buffer requirements in percentage of video size and the playback ratio $\lfloor\alpha\rfloor$ on two channels, and it compares the average and maximum buffer size of RFB (AvgRFB, MaxRFB), RAB (AvgRAB, MaxRAB), HRAB normal (AvgNor, MaxNor)

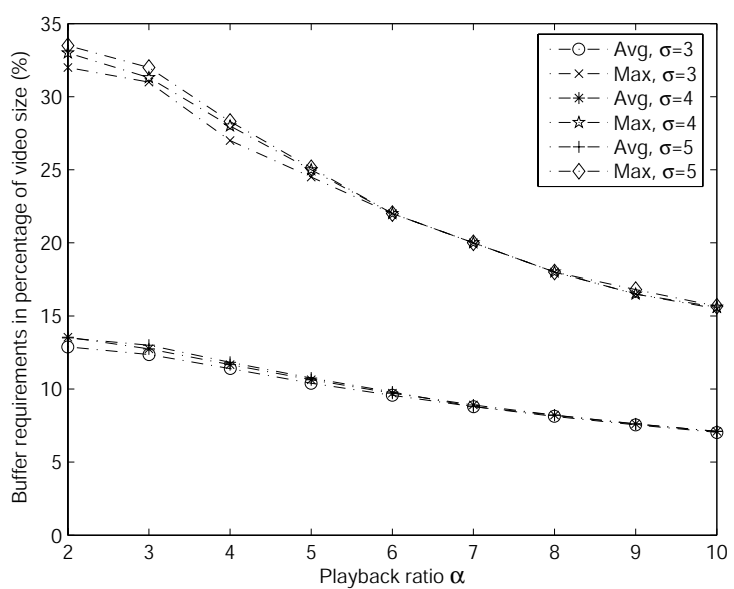

Figure 5: Comparison of required buffers of the HRAB normal case.

and the worst (AvgWor, MaxWor) case $(\sigma=2)$. As shown in the figure, the average and maximum buffer requirements of RFB is much larger than RAB and HRAB because RFB will not cut more segments as $\lfloor\alpha\rfloor$ increased. For the maximum value, MaxRFB will need $33 \%$ of a playing video. The required buffer ranges of MaxRAB, MaxNor and MaxWor are $15 \%$ to $26 \%, 15 \%$ to $31 \%$, and $8 \%$ to $18 \%$. The MaxNor will use more buffer than MaxRAB since the channel transmission rate used by HRAB is higher than RAB, so it will waste more buffer in downloading $s_{0}$. For an average value, AvgRFB and AvgNor need buffer ranges of $14 \%$ to $19 \%$ and $7 \%$ to $12 \%$. AvgRAB and AvgWor can both be lowered to below 10\%. Figure 5 and 6 depict the normal and worst case of HRAB when $\sigma$ increase the needed buffer size. In this case, it will not increase substantially needed buffer requirement as $\sigma$ increasing, and the download time $T_{d}^{\prime}$ will decrease to $1 / \sigma$ while $\sigma$ increasing. For the maximum value, the required buffer ranges of $\sigma=3$ to 5 are $16 \%$ to $33 \%$ in the normal case and $8 \%$ to $22 \%$ in worst case. For the maximum value, the required buffer ranges of $\sigma=3$ to 5 are $7 \%$ to $14 \%$ in the normal case and $4 \%$ to $12 \%$ in worst case.

To compare RAB, HRAB, RFB, and DBB of a 7200s video length, the initialization average waiting time are shown in Figure 7. In the RFB scheme the range of waiting time is from 120 to $600 \mathrm{~s}$. In the DBB scheme, to use the best average waiting time to compare, the range of waiting time is from 144 to 1440s. The RAB and HRAB each needs 31 to 417 s minimum waiting time (depending of the tolerable subscriber's waiting time $\rho$ ). In the HRAB, worst case can achieve the minimum buffer requirement and minimum waiting time, but it will cause a heavier burden to the system. Referring to (8) the probability of the worst case can be calculated, shown in table 3 .

Table 3: Probability in worst case

\begin{tabular}{llllll}
\hline Playback Ratio $(\lfloor\alpha\rfloor)$ & 1 & 2 & 3 & 4 & 5 \\
Probability (\%) & 49.79 & 32.96 & 24.47 & 19.33 & 15.85 \\
\hline \hline Playback Ratio $(\lfloor\alpha\rfloor)$ & 6 & 7 & 8 & 9 & 10 \\
Probability (\%) & 13.33 & 11.40 & 9.87 & 8.61 & 7.57 \\
\hline
\end{tabular}




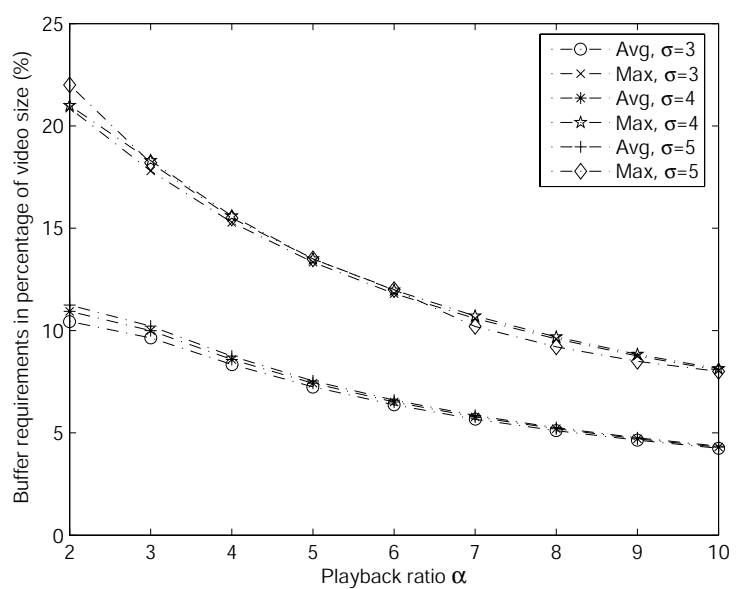

Figure 6: Comparison of required buffers of the HRAB worst case.

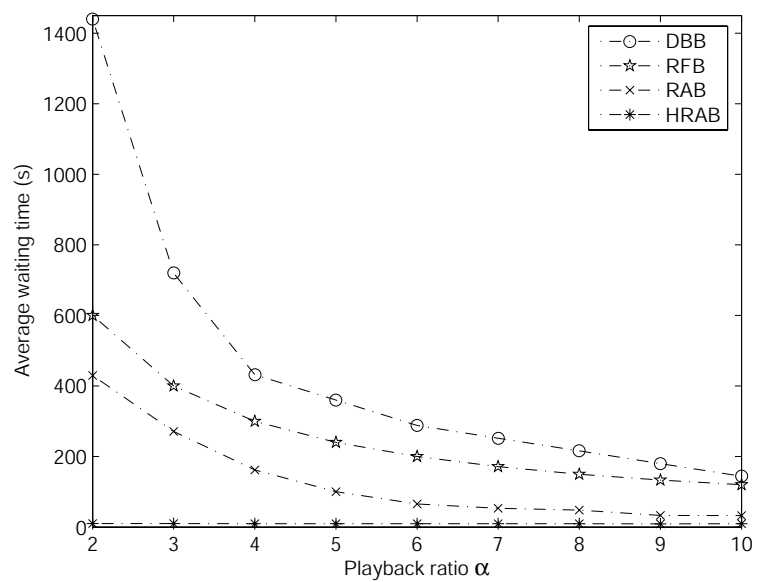

Figure 7: Average waiting time.

\section{CONCLUSIONS}

This paper proposes the RAB and HRAB schemes to reduce the size of required buffer and the waiting time of scribers in the WiMAX-VoD system according to the parameter $\alpha$, which is obtained from the ratio of download rate and video playing rate. Simulation results show that RAB and HRAB can efficiently decrease the required size of buffering under $10 \%$ of the size of video and reduce the waiting time as lower as possible for subscribers. Specifically, since it only require the subscriber to maintain a lower buffer size, it is very suitable for applications in mobile devices. Moreover, by considering the mobility of MSSs, the RAB can be investigated further for supporting $\mathrm{VoD}$ broadcasting among mobile wireless network. In the future, power saving framework and current VoD broadcasting system will be added into the system for further analysis and comparison.

\section{REFERENCES}

[1] H.-F. Yu., H.-C. Yang, and L.-M. Tseng "Reverse Fast Broadcasting (RFB) for Video-on-Demand
Applications," IEEE Trans. Broadcasting, vol. 53, no. 1, pp. 103-111, March 2007.

[2] T. Yoshihisa, M. Tsukamoto, and S. Nishio, "A Scheduling Scheme for Continuous Media Data Broadcasting with Prefetching," IEEE Trans. Broadcasting, vol. 52, no. 1, pp. 1-10, March 2006.

[3] K.-A. Hua, M.-A. Tantaoui, and W. Tavanapong, "Video Delivery Technologies for Large-Scale Deployment of Multimedia Applications," Proc. IEEE, vol. 92, no. 9, pp. 1439-1451, sept. 2004.

[4] A. Hu, "Video-on-Demand Broadcasting Protocols: A Comprehensive Study," in Proc. IEEE INFOCOM 2001, vol. 1, pp. 508-517, Alaska, April 2001.

[5] J.-P. Sheu, H.-L. Wang, C.-H. Chang, and Y.-C. Tseng, "A Fast Video-on-Demand Broadcasting Scheme for Popular Videos," IEEE Trans. Broadcasting, vol. 50, no. 2, pp. 120-125, June 2004.

[6] C.-T. Lin, and J.-W. Ding, "CAR: A Low Latency Video-on-Demand Broadcasting Scheme for Heterogeneous Receivers," IEEE Trans. Broadcasting, vol. 52, no. 3, pp. 336-349, Sept. 2006.

[7] Y.-C. Tseng, M.-H. Yang, and C.-H. Chang, "A Recursive Frequency-Splitting Scheme for Broadcasting Hot Videos in VoD Service," IEEE Trans. Commun., vol. 50, no. 8, pp. 1348-1355, Aug. 2002.

[8] M.-A. Tantaoui, K.-A. Hua, and T.-T. Do, "BroadCatch: A Periodic Broadcast Technique for Heterogeneous Video-on-Demand," IEEE Trans. Broadcasting, vol. 50, no. 3, pp. 289-301, Sept. 2004.

[9] A. Dan, D. Sitaram, and P. Shahabuddin, "Scheduling Policies for An on-Demand Video Server with Batching," in Proc. 2nd ACM Int. Conf. Multimedia, pp. 15-23, Oct. 1994.

[10] A. Dan, D. Sitaram, and P. Shahabuddin, "Dynamic Batching Policies for An on-Demand Video Server," in Proc. ACM Multimedia Syst., vol. 4, pp. 112-121, June 1996.

[11] IEEE 802.16 Working Group, "IEEE Standard for Local and Metropolitan Area Networks Part 16," IEEE Std. 802.16e-2005, Feb. 2006.

[12] WiMAX Forum Working Group, "WiMAX Forum Mobile System Profile 3," WiMAX Forum Release 1.0, Approved Specification, Revision 1.2.2, Nov. 2006.

[13] S. Choi, G.-H. Hwang, T. Kwon, A.-R. Lim, and D.-H. Cho "Fast Handover Scheme for Real-Time Downlink Services in IEEE 802.16e BWA System," in Proc. IEEE VTC 2005-Spring, vol. 3, pp. 2028-2032, Dallas, Texas, June 2005.

[14] J. Chen, et al., "The Design and Implementation of WiMAX Module for ns-2 Simulator," in Proc. ACM VALUETOOLS 2006, article 5, Pisa, Italy, Oct. 2006.

[15] A. Ksentini, A. Gueroui, and M. Naimi, "Improving H.264 Video Transmission in 802.11e EDCA," in Proc. IEEE ICCCN 2005, pp. 381-386, California, USA, Oct. 2005.

[16] The video file comparison. http://www.videohelp.com/comparison.htm. 